\title{
Personage Column: Helmut Friess
}

Professor Helmut Friess is the Vice Chairman of the Department of General Surgery, University of Heidelberg, Germany and heads the European Pancreas Center for clinical and basic research in pancreatic disorders.

In the early 90's he did his research fellowship in the laboratory of Professor Murray Korc in California, USA, where he made significant contributions in the understanding of pancreatic diseases at the molecular level. Later he became consultant surgeon in the Department of Visceral Surgery, University of Bern, Switzerland under the chairmanship of Professor Markus W. Büchler.

Among others, he is a member of the European Pancreatic Club, New York Academy of Medical Sciences, American Pancreatic Association, European Pancreas Club, European Society for Surgical Research, European Digestive Surgery, and International Hepato-Pancreato-Biliary Association.

$\mathrm{He}$ is an associate editor of Digestive Surgery and is on the editorial board of many journals including Pancreatology and Langenbecks Archives of Surgery.

He has been invited for numerous lectures, is a visiting professor to universities worldwide, and has published over 300 original articles and written more than 80 book chapters.

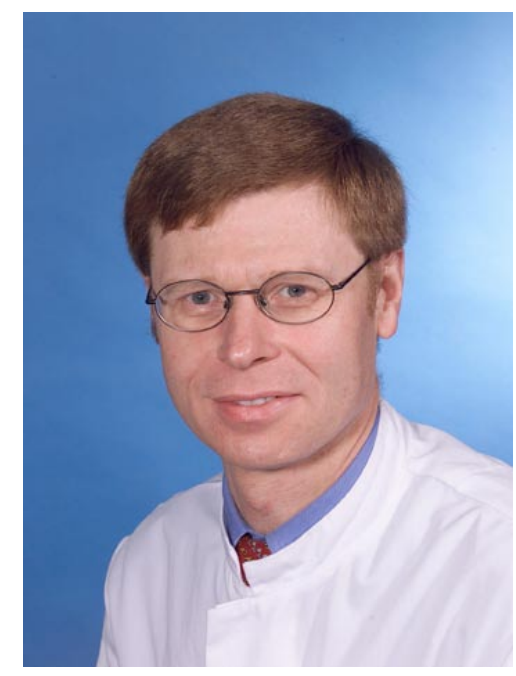

Helmut Friess, M.D.

Department of General Surgery

University of Heidelberg

Im Neuenheimer Feld 110

69120 Heidelberg, Germany

Phone: + 496221564860

Fax: + 496221566903

Email:helmut_friess@med.uni-heidelberg.de 